\title{
Distance-based separability criterion of ROI in classification of farmland hyper-spectral images
}

\author{
Tang J inglei ${ }^{1,2}$, Miao Ronghui ${ }^{3}$, Zhang Zhiyong ${ }^{1 *}$, Xin J ing ${ }^{2}$, Wang Dong ${ }^{1}$ \\ (1. College of Information Engineering, Northwest A\&F University, Yangling 712100, Shaanxi, China; 2. Shaanxi Key Laboratory of \\ Complex System Control and Intelligent Information Processing, Xi'an University of Technology, Xi'an 710048, China; \\ 3. College of Information Science and Engineering, Shanxi Agricultural University, Taigu 030801, Shanxi, China)
}

\begin{abstract}
The hyper-spectral image contains spectral and spatial information, which increases the ability and precision of objects classification. Despite the classification value of hyper-spectral imaging technology within various applications, users often find it difficult to effectively apply in practice because of the effect of light, temperature and wind in outdoor environment. This research presented a new classification model for outdoor farmland objects based on near-infrared (NIR) hyper-spectral images. It involves two steps including region of interest (ROI) acquisition and establishment of classifiers. A distance-based method for quantitative analysis was proposed to optimize the reference pixels in ROI acquisition firstly. Then maximum likelihood (ML) and support vector machine (SVM) were used for farmland objects classification. The performance of the proposed method showed that the total classification accuracy based on the reference pixels was over $97.5 \%$, of which the SVM-M model could reach 99.5\%. The research provided an effective method for outdoor farmland image classification.
\end{abstract}

Keywords: distance-based separability criterion, near-infrared hyper-spectral image, ROI, farmland image classification DOI: $10.25165 /$ j.ijabe.20171005.2264

Citation: Tang J L, Miao R H, Zhang Z Y, Xin J, Wang D. Distance-based separability criterion of ROI in classification of farmland hyper-spectral images. Int J Agric \& Biol Eng, 2017; 10(5): 177-185.

\section{Introduction}

During working in farmland, intelligent agricultural robots should firstly perceive environmental information with sensors, and then decide the walking strategy and the direction $^{[1]}$. Since the farmland environment has the characteristics of openness, unstructured and complex scenes, it would lead to information uncertainty when

\section{Received date: $2015-12-10 \quad$ Accepted date: $2017-03-01$}

Biographies: Tang Jinglei, PhD, Associate Professor, research interest: computer vision, image processing, Email: tiana_tang@ 163.com; Miao Ronghui, Master, research interest: image processing, Email: ronghui092@163.com; Xin Jing, PhD, Professor, research interest: target recognition and tracking, Email: xinj@xaut.edu.cn; Wang Dong, Bachelor, research interests: computer vision, image processing. Email: dongwang@ nwsuaf.edu.cn.

*Corresponding author: Zhang Zhiyong, $\mathrm{PhD}$, Associate Professor, research interests: robot control, target recognition and tracking. No.3 Taicheng Road, Yangling 712100, Shaanxi, China. Tel: +86-15229333257, Email: zzy@nwsuaf. edu.cn. intelligent agricultural robots perceive the environmental information ${ }^{[2]}$. Therefore, the classification for various farmland objects is of great significance in environment perceiving. At the same time, hyper-spectral imaging technology is the combination of traditional imaging and spectroscopy technology ${ }^{[3]}$. Compared with traditional images, the hyper-spectral image contains abundant spectral and spatial information. In addition, hyper-spectral image provide hundreds of narrow and contiguous spectral bands with a fine spectral resolution which have been widely used in military, medicine, agriculture, environmental monitoring and other fields ${ }^{[4]}$.

Although hyper-spectral imaging technology has been widely used in agriculture, there are limitations. In indoor research, most of the detections aim at single agricultural products. ElMasry et al. ${ }^{[5]}$, Cai et al.$^{[6]}$ and Cheng et al. ${ }^{[7]}$ applied hyper-spectral imaging technology to apple freezing damage, citrus fruit rust detection and apple size prediction. The main reasons are: easy 
accessibility for single agricultural products; low requirements for indoor environment and equipment; easy separation of background with agricultural products. In outdoor research, hyper-spectral images are mostly applied in aerial remote sensing. Ghamisi et al. ${ }^{[8]}$ proposed a new method which is based on combining hidden Markov random field segmentation with SVM classifier, and in order to preserve edges in the final classification map, a gradient step is taken into account. Liao et al. ${ }^{[9]}$ proposed a novel semi-supervised local discriminant analysis method for feature extraction in hyperspectral remote sensing imagery, with improved performance in both ill-posed and poor-posed conditions. Tarabalka et al. ${ }^{[10]}$ proposed a method using a probabilistic support vector machine for hyperspectral image classification, in which a Markov random field regularization is adopted for refining the classification results. Moreover, Tarabalka et al. ${ }^{[11]}$ computed the robust color morphological gradient of the hyperspectral image, and then applied the watershed transformation to the obtained gradient. However, with atmospheric radiation and severe distortion, the aerial remote sensing images cannot meet the demands for near-earth agricultural robot operation. Furthermore, based on the high requirements for instruments and equipment, outdoor environment is vulnerable to the influence of factors such as light, temperature and wind. Therefore, in this study the hyper-spectral imaging technology was applied to outdoor near-earth farmland, so as to promote the operations of agricultural robots in farmland environment with high credibility.

Farmland hyper-spectral images include the image and spectral information of objects. Through observations, the main farmland objects are crops, roads, trees and soil, etc., and these objects can be represented by a certain specific spectrum. Thus, the classification of farmland objects by spectrum analysis technique is feasible.

In this research, intelligent agricultural robot takes the near-infrared hyper-spectral farmland images as the research objects. It adopts spectral analysis and spectral pre-processing for farmland objects extraction and classification. Through the processes of image de-noising, feature extraction, image classification and post-processing, a classification model about farmland objects was put forward. The overall classification accuracy and Kappa coefficient were adopted to validate the classification results, and the distributions of various farmland objects were analyzed. On the basis of studying farmland image classification, an environment perception mechanism can be built for farmland images.

\section{Image acquisition and preprocessing}

\subsection{Equipment}

HyperSIS series of agricultural hyper-spectral imaging system was designed specifically for agricultural products based on N17E imaging spectrometer which was imported from Finland, and this system can be equipped with indoor and outdoor coverings scan accessories. In this study, the outdoor coverings scan accessories was used to obtain hyper-spectral images. The system consisted of push-broom imaging spectrometer (N17E), array detectors, SpectralSENS data acquisition software, light source and outdoor coverings scan accessories, and other components. The type of N17E imaging spectrometer is bulk phase holographic transmission grating: the wavelength range is $900-1700 \mathrm{~nm}$, and the spectral resolution is $5 \mathrm{~nm}$, the spectral sampling point is $4 \mathrm{~nm}$. The full-frame pixel number of the NIR array detectors is $320 \times 256$. The RSA series electronically controlled rotary table was used as outdoor measuring accessories. The SpectralSIS software was used for spectra and real-time image acquisition. It is diffused light source of white light with the spectral range of $350-2500 \mathrm{~nm}$ and the power of more than $100 \mathrm{~W}$.

\subsection{Spectral data acquisition}

Near-infrared hyper-spectral images of soybean fields were selected as research targets, and the main objects included crops, roads, trees and soil. The images were taken in the agriculture experimental fields of Northwest A\&F University North Campus, after shooting span of 30 d, 96 samples were obtained under different time, temperature, light and weather conditions, and each of them had 256 bands. After analyzing the spectral curves of the above samples, 11 outliers were removed, and then the remaining 85 images were selected for subsequent researches. 
Before collecting the hyper-spectral image data, it needs to set parameters of the system in order to get a clear collected image, and adjust the conveyor speed to avoid resolution distortion of the image ${ }^{[3]}$. Hyper-spectral camera parameters were set as follows: the exposure time was $10 \mathrm{~ms}$; the moving speed of the electronically controlled displacement table was $20 \mathrm{~mm} / \mathrm{s}$; the hyper-spectral image size was $320 \times 400 \times 256$. The acquisition of hyper-spectral image data was based on GILDEN Potonic SpectraSENS 3.1.1-Xenics $\times 32$ data acquisition software platform, and the analysis and processing of hyper-spectral image data was based on ENVI4.7 software platform.

During data acquisition, each captured image is numbered. The collected images contained both the image information and the spectral information, which were three-dimensional data. In order to express the spectral characteristics of each pixel, a two-dimensional spectral curve was introduced. Figure 1 shows the image information and spectral information of $1226 \mathrm{~nm}$ band, and Figure 2 shows the classification process of farmland objects. It consisted of image acquisition, spectral preprocessing, and farmland objects classification.

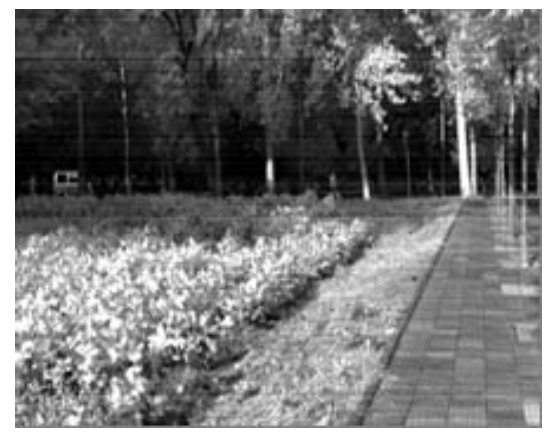

a. Image information of $1226 \mathrm{~nm}$ band

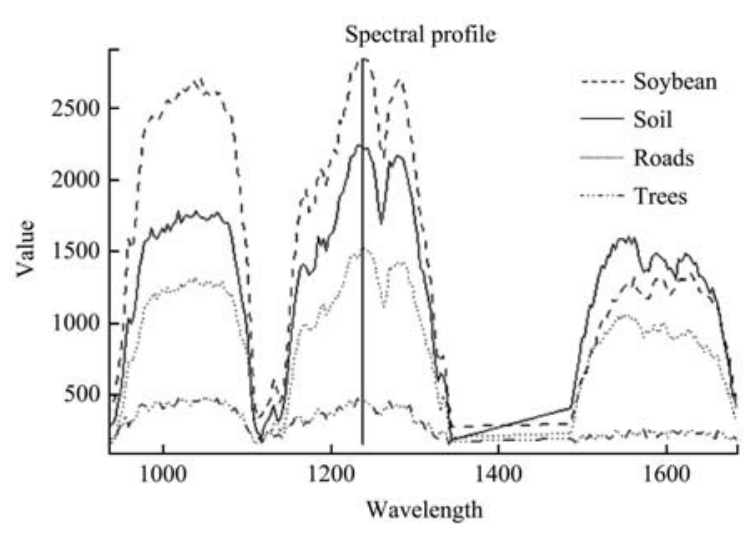

b. Spectral information of $1226 \mathrm{~nm}$ band

Figure 1 Image information and spectral information of $1226 \mathrm{~nm}$ band

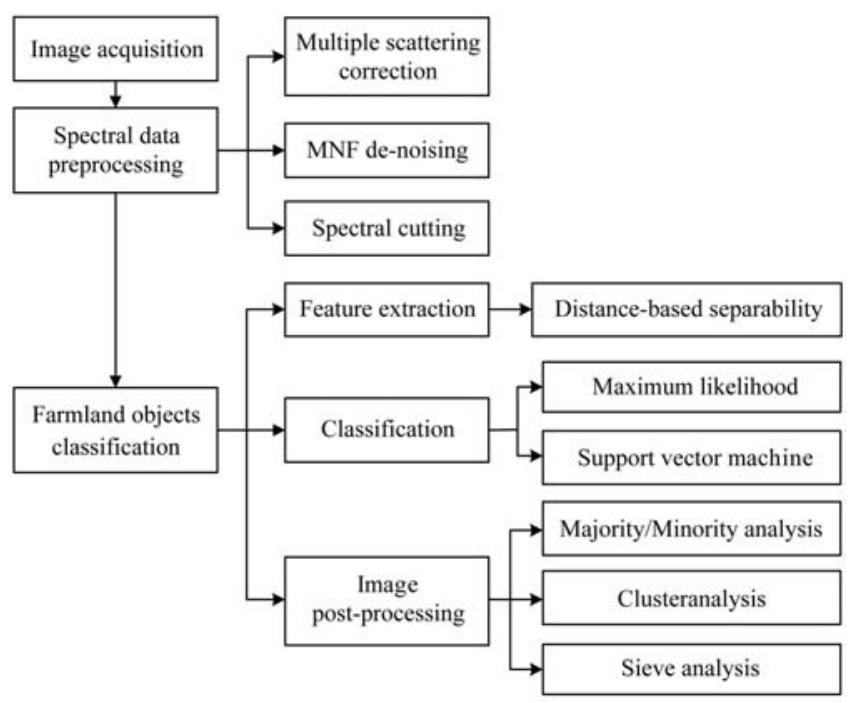

Figure 2 Classification process of farmland objects

\subsection{Spectral data preprocessing}

Spectral data preprocessing mainly includes two aspects: one is to eliminate the influence of noise, and the interference of background and measurement devices. The second is to optimize the spectral ranges in order to obtain effective spectral regions.

Considering the impact factors such as instrument and background, the main preprocessing methods were de-noising, radiation preprocessing (scaling) and geometric correction. Analysis shows that there are 'Bad lines' and stripe noise in the hyper-spectral farmland images. Therefore, calibration and de-noising processing are needed. 'Bad line' is the inequality of radiation brightness when the images are acquired by sensor, then multiple scattering corrections for image correction can effectively overcome this issue. Stripe noise is removed by using minimum noise fraction $(\mathrm{MNF})^{[12]}$, in which the noise can be separated from the images. In geometric correction, for airborne aerial hyper-spectral remote sensing images, the linear CCD sensor can be easily affected by airstream, which would lead to a drastic attitude changes and serious image geometric distortion. However, the images obtained in this study are near-earth hyper-spectral images, and there is almost no atmospheric reflection deviation, so geometric correction is not being considered. In spectral optimization, spectral cutting is adopted for analysis.

\subsubsection{Multiple scattering correction}

In the process of image acquisition, storage, processing and transmission, the unevenly distributed of 
light intensity in each band, different surface shape of each farmland object, lead to the large noise among the weaker light intensity distribution bands. Therefore, the acquired images must be corrected in order to eliminate the noise. The SpectraSENS data acquisition software has the function of self-correction, and the self-correction function was adopted to remove the noise.

\subsubsection{MNF de-noising}

In the process of image acquisition, noises would emerge by influences of various factors, among which stripe noise is one of the most obvious. It is a special kind of noise presenting with certain periodicity, directivity and zonal distribution in the images. This noise is mainly caused by the scanning probe response differences, the sensors mechanical movement, and the temperature changes in the process of scanning and imaging of the sensor light and electric parts ${ }^{[13]}$.

Minimum noise separation technology (MNF) can separate noise from the hyperspectral images and determine the intrinsic characteristics dimension of image data $^{[14]}$. Thus, in this research we adopted MNF for image de-noising. The contrast images before and after stripe noise removal are shown in Figure 3. By de-noising, the stripe noise can be eliminated effectively.

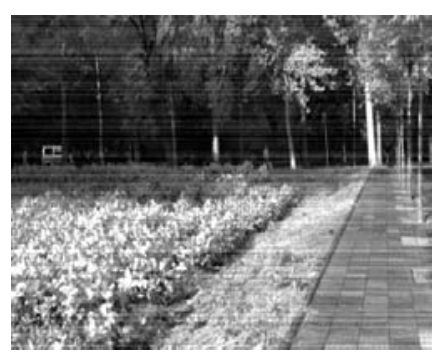

a. Before de-noising

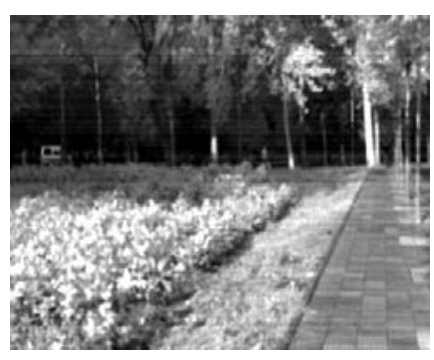

b. After de-noising
Figure 3 Contrast images before and after stripe noise removal

\subsubsection{Spectral cutting}

Hyper-spectral image cutting includes spatial and spectral cuttings. Due to the fact that the acquired farmland images have complex background and various objects, and the image itself is $320 \times 400$, then spatial cutting would against farmland object extraction. The band range of the images collected by the hyper-spectral imaging system is 865.11-1711.11 nm. And spectral cutting can reduce the amount of hyper-spectral image data and provide convenience for subsequent analysis.

Figure 4 shows the spectral curve of a hyper-spectral image region. From Figure 4, it can be seen that large noise existed at 865.11-933 nm, 1344-1484 $\mathrm{nm}$ and 1686-1711.11 $\mathrm{nm}$ range, and the image information at these bands is fuzzy and useless, so the spectral images were cut and 934-1343 nm and 1485-1685 nm of 185 bands images retained.

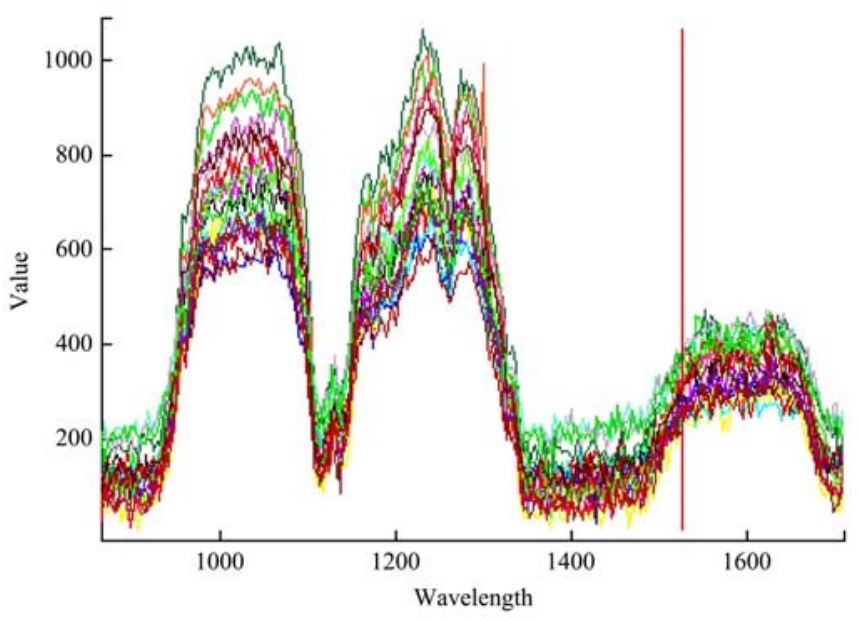

Figure 4 Spectral curve of a hyper-spectral image region

\section{Classification farmland objects based on region of interest (ROI)}

$\mathrm{ROI}$ is a region which can arouse the interests of users and best represent the image content. The regions selected by users are visually important areas, which would be more conforming to human subjective evaluation $^{[15]}$. Therefore, this study adopts ROI for feature vectors extraction.

\subsection{Feature extraction}

Feature extraction is one of the most important and difficult parts in pattern recognition, and analyzing the effectiveness of various features and choosing the most representative characteristics are also the key steps. And the effectiveness of the extracted features will directly determine the classification results ${ }^{[16]}$. Hence, feature extraction becomes the primary problem in classification. In this paper, reference pixels are obtained based on ROI at first, and then optimized using quantitative analysis method. Generally, the reference pixels are accounted for $10 \%$ of the whole image. In view of the global farmland objects, multiple irregular areas are selected for each object as the reference points. The images include crops, roads, trees, and soil, besides that, the remaining parts will be regarded as background. 
A number of reference points of the five classes are selected randomly. As shown in Figure 5, the red, yellow, blue, green and magenta areas represent the crops, roads, trees, soil, and the background respectively.

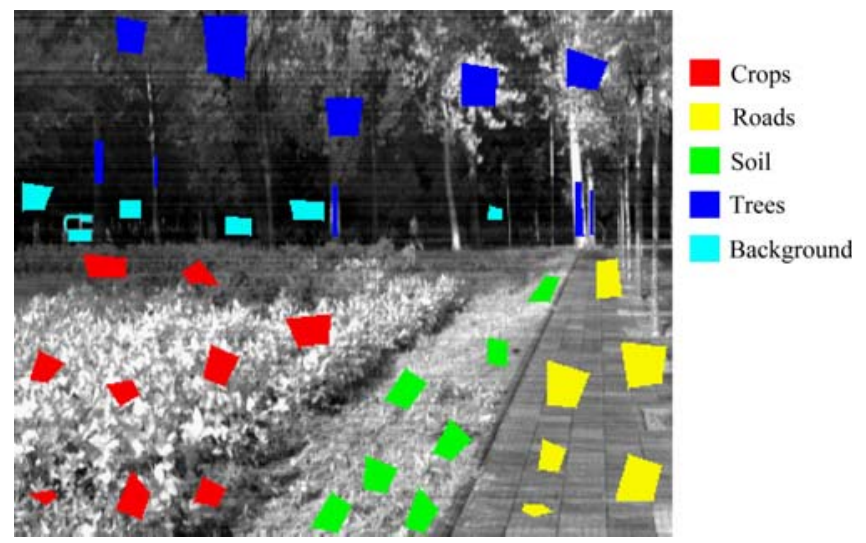

Figure 5 Reference pixels

Quantitative analysis is implemented by calculating the separability of different objects to determine stand or fall of the classification result. Commonly used separability criterions are mainly based on distance, probability distribution and entropy function. In this study, we put forward a distance-based separability criterion to measure the effectiveness of the classification, which is to determine the separability between classes by calculating the average distance of all samples. Assuming that the total number of the classes is $\mathrm{c}$, and the distance of point to point-sets is expressed by the average Euclidean distance. The number of each class is $n_{i}$, $i=1,2, \ldots, c, x_{k}^{(i)}$, represents the $k$ vector of class $i, k=1$, $2, \ldots, n_{i}$. The separability can be expressed in the distance of within-class and between-class. The distance of with-class is the mean square distance between each samples in class $i$. The distance of between-class is the mean square distance between each class. To obtain the distance of within-class, the average vector for each class is needed and calculated by Equation (1).

$$
m_{i}=\frac{1}{n_{i}} \sum_{k=1}^{n_{i}} x_{k}^{(i)}
$$

Then the distance of within-class is calculated by Equation (2).

$$
d\left(w_{i}\right)=\frac{1}{n_{i}} \sum_{k=1}^{n_{i}} \delta\left(x_{k}^{(i)}, m_{i}\right)
$$

which, $i=1,2, \ldots, c, w_{i}$ is the within-class distance vector.
To obtain the distance of between-class, the average vector of all classes is expressed as Equation (3).

$$
m=\sum_{i=1}^{c} p_{i} m_{i}
$$

where, $P_{i}$ is the prior probability of each class.

Then the distance of between-class is calculated by Equation (4).

$$
d\left(b_{i}\right)=\delta\left(m_{i}, m\right)
$$

which, $i=1,2, \ldots, c, b_{i}$ is the between-class distance vector.

Combining the distance of within/between-class, we can obtain the class separability with Equation (5).

$$
J_{d}(x)=\sum_{i=1}^{c} P_{i}\left[d\left(w_{i}\right)+d\left(b_{i}\right)\right]=\sum_{i=1}^{c} P_{i}\left[\frac{1}{n_{i}} \delta\left(x_{k}^{(i)}, m_{j}\right)+\delta\left(m_{i}, m\right)\right]
$$

which,

$$
\sum_{i=1}^{c} P_{i} \delta\left(m_{i}, m\right)=\frac{1}{2} \sum_{i=1}^{c} P_{i} \sum_{j=1}^{c} P_{i} \delta\left(m_{i}, m_{j}\right)
$$

According to Equation (5), the average distance of all samples is expressed as Equation (7).

$$
J_{d}(x)=\frac{1}{2} \sum_{i=1}^{c} P_{i} \sum_{j=1}^{c} P_{j} \frac{1}{n_{i} n_{j}} \sum_{k=1}^{n_{i}} \sum_{l=1}^{n_{j}} \delta\left(x_{k}^{(i)}, x_{l}^{(j)}\right)
$$

On the premise of Euclidean distance is calculated by Equation (8).

$$
\delta\left(x_{k}^{(i)}, x_{l}^{(j)}\right)=\left(x_{k}^{(i)}-x_{l}^{(j)}\right)^{T}\left(x_{k}^{(i)}-x_{l}^{(j)}\right)
$$

With the distance matrices, it is convenient to calculate and express the spatial distribution of the samples. Here, the between-class discrete matrix is expressed as Equation (9).

$$
\tilde{S}_{b}=\sum_{i=1}^{c} P_{i}\left(m_{i}-m\right)\left(m_{i}-m\right)^{T}
$$

The within-class discrete matrix is calculated by Equation (10).

$$
\tilde{S}_{w}=\sum_{i=1}^{c} P_{i} \frac{1}{n_{i}}\left(x_{k}^{(i)}-m_{i}\right)\left(x_{k}^{(i)}-m_{i}\right)^{T}
$$

The class separability in matrix trace is expressed as Equation (11):

$$
J_{d}(x)=\operatorname{tr}\left(\tilde{s}_{b}+\tilde{s}_{w}\right)
$$

When using the separability criterion for classification, the coefficient should be normalized. If the coefficient is more than 0.9 , it means there are big differences between the classes, and the selected samples are 
appropriate. If the coefficient is between 0.9 and 0.7 , the ROI need to be modified until the results can meet requirements. If the coefficient is less than 0.7 , it means there is less difference between the classes, and the classes should be merged. The regions of interest are modified based on quantitative analysis, and reference pixels as shown in Table 1.

Table 1 Reference pixels

\begin{tabular}{lcccccc}
\hline \multicolumn{1}{c}{ Reference pixels } & Crops & Roads & Trees & Soil & Background & Total \\
\hline Before quantitative analysis & 2458 & 2125 & 2989 & 2045 & 1034 & 10651 \\
After quantitative analysis & 2302 & 2345 & 3128 & 1962 & 947 & 10684 \\
Regions & 9 & 6 & 10 & 7 & 9 & 41 \\
\hline
\end{tabular}

\subsection{Classifier establishment}

Despite the image classification value of maximum likelihood (ML) ${ }^{[17]}$ and support vector machine $(\mathrm{SVM})^{[18]}$ with various applications, users often find it difficult to effectively apply ML techniques and SVM techniques in establishment of classifier. Moreover, ML is a typical supervision classifier based on statistical analysis according to the Bayes rules ${ }^{[19]}$. SVM is a machine learning method based on statistical learning theory ${ }^{[20]}$, and it follows the principle of minimum structure risk, which can be a good solution to small samples, nonlinear, high dimension and local Minimization ${ }^{[21]}$. This study adopts SVM and ML for image classification based on these advantages.

As SVM is a typical two-class classifier, we need to establish multi-class classifiers to achieve recognition of farmland objects. Compared with one-against-one (OAO) method, in the process of solving optimization problems, one-against-all (OAA) method would produce more variables, and it can also cause slow training speed. Therefore, OAO is used to establish the multi-class classifiers.

On parameter selection, $C$ and $\gamma$ are two necessary parameters. They are the punish coefficient parameter and the interval, respectively, which would directly affect the classification accuracy. Parameters estimation of $C$ and $\gamma$ are based on cross-validation and grid -search method, and using the parameter selection model of Grid. Py to search them ${ }^{[22]}$. Here $\gamma=0: 004$ and $C=0: 25$. The kernel function of SVM is radial basis function (RBF) as $12^{[23]}$.

$$
K(x, y)=\exp \{-\gamma\|x-y\|\}
$$

The implementation of ML is as follows.

(1) The means, variances and co-variances of the classes can be calculated according to the training sets. Assuming that the distribution of each class conforms multi-dimensional normal distribution. Thus, conditional probability density function is calculated by Equation (13).

$$
P\left(x / y_{i}\right)=(2 \pi)^{-\frac{n}{2}}\left|\sum i\right|-\frac{1}{2} \exp \left[\frac{1}{2}\left(x-\mu_{i}\right)^{T} \sum i^{-1}\left(x-\mu_{i}\right)\right]
$$

which, $x$ and $\mu_{i}$ represent the mean vector and covariance matrix of $y_{i}$, respectively; $n$ is band number.

(2) According to Bayes decision function, posterior probability is calculated by Equation (14).

$$
P\left(y_{i} / x\right)=\frac{P\left(x / y_{i}\right) P\left(y_{i}\right)}{P(x)}
$$

which, $P\left(y_{i}\right)$ is the prior probability of $y_{i} ; P(x)$ is a public item which is irrelevant to the class.

According to the discrimination function-maximum posterior probability principle, the type of the pixel can be determined. If $p\left(y_{i} / x\right)=\max _{j}^{C}\left(p\left(y_{i}\right) / x\right)$, then $x$ belongs to $y_{i}$.

Figure 6 shows the classification results of SVM and ML. It can be seen from the figure that the classification result of SVM seems to be better, and the farmland objects are clearly separated, which conform to human eye visual perception results. While, the ML does not perform well, there are obvious confusions in different levels among the crops, soil and roads. This is mainly caused by the fact that there is too much weed in the soil, causing the confusion of crops and weeds. The boundary of crops, soil and roads is fuzzy, resulting in a serious connecting phenomenon.

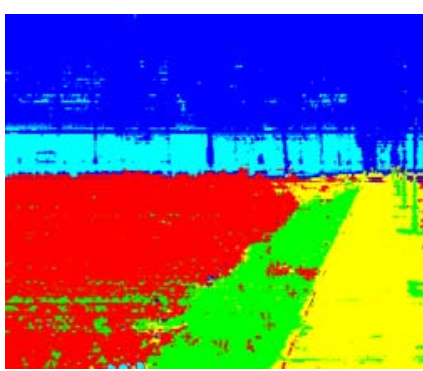

a. Classification result of SVM

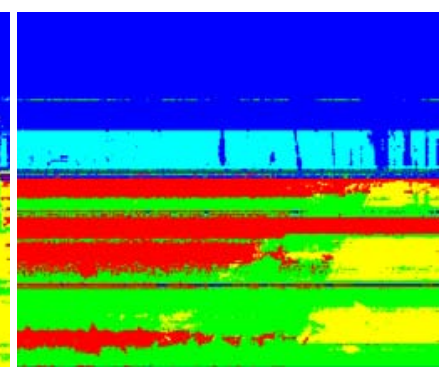

b. Classification result of ML
Figure 6 Classification results of SVM and ML 


\subsection{Image post-processing}

Obvious salt-and-pepper noise and isolated points exist in the images after classified by ML and SVM, and then so post-processing is needed. In this paper, majority/minority analysis, cluster analysis and sieve analysis were respectively used for post-processing, and all these three methods are effective ways to optimize the classification results, by using these we can correct the wrong locations and remove the salt-and-pepper noise and isolated points.

Majority/Minority analysis is realized by using a $3 \times 3$ convolution template to conduct translational convolution operation. Majority analysis is to use the largest classes replace the smallest classes, and minority analysis is the opposite. Cluster analysis is also realized by using a $3 \times 3$ convolution template to conduct translational convolution operation, and then clusters the same classes. Sieve analysis consists in deleting a class by setting the minimum number of pixels. Here, we set 2 as the minimum threshold. When the classification pixels are less than 2, the class would be eliminated. The classification results of the three methods are shown in Figure 7. It can be seen that the results of majority/minority and cluster analysis work well, but the sieve analysis makes more isolated points, so its effect is worse.

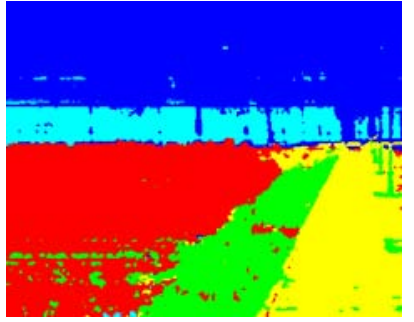

a. SVM-M

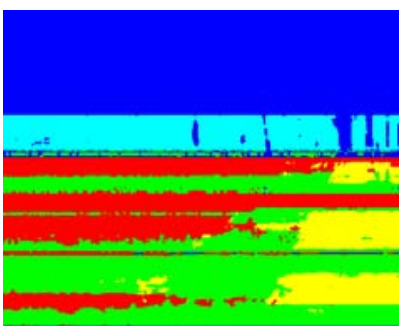

d. ML-M

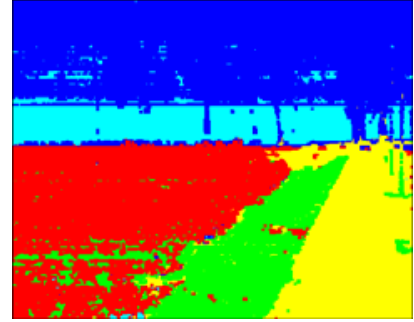

b. SVM-C

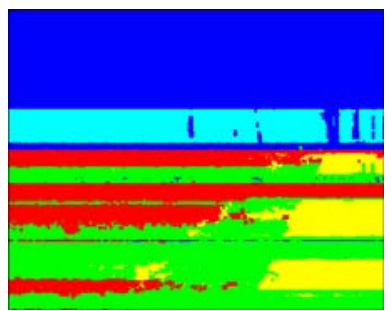

e. ML-C

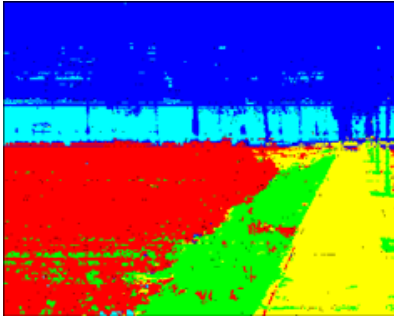

c. SVM-S

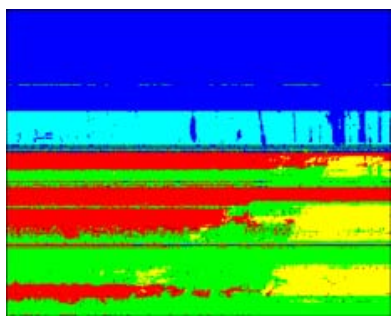

f. ML-S

Figure 7 Classification results of the three methods

\section{Results and discussion}

The classification results were mainly analyzed from the following three aspects: the specific classification results of different farmland objects, the classification results of different classification methods, and the distributions of different farmland objects. The processor of this experiment is dual-core @ $4.0 \mathrm{GHz} \mathrm{CPU}$ (Intel core i7-6700K) with $128 \mathrm{G}$ memory. These reference pixels are partitioned into testing sets and training sets by $K$-fold cross-validation $(K=10)$. The original reference pixels are randomly partitioned into 10 subsets, and a single subset is retained as the validation data for testing, and the remaining 9 subsets are used as training data. The cross-validation process is repeated 10 times, with each of the 10 subsets used exactly once as the validation data ${ }^{[23]}$.

In this study, the SVM classification results were selected to conduct specific analysis, as shown in Table 2 . Table 2 clearly shows the specific pixels of right and wrong classifications for different farmland objects. The main reasons for the wrong classification are that the farmland environment is complex and there is no clear boundary between different objects. It also can be seen that all the classification accuracies are extremely high.

Table 2 SVM classification results

\begin{tabular}{ccccccc}
\hline $\begin{array}{c}\text { Classification } \\
\text { results }\end{array}$ & Crops & Soil & Trees & Roads & Background & Total \\
\hline Crops & 2286 & 42 & 0 & 0 & 0 & 2328 \\
Soil & 14 & 1914 & 0 & 6 & 0 & 1934 \\
Trees & 0 & 0 & 3116 & 6 & 12 & 3128 \\
Roads & 2 & 6 & 0 & 2339 & 1 & 2348 \\
Background & 0 & 0 & 12 & 0 & 934 & 946 \\
Total & 2302 & 1962 & 3128 & 2345 & 947 & 10684 \\
\hline
\end{tabular}


The classification evaluation is measured by the overall classification accuracy and Kappa coefficient. Table 3 is the classification accuracy by different methods, in which SVM-M, SVM-C and SVM-S respectively stand for the results by majority/minority analysis, cluster analysis and sieve analysis on the SVM image, and the same as ML-M, ML-C, and ML-S. From the table, it can be seen that the overall classification accuracy by the method proposed in this research has reached more than $97.5 \%$, of which the overall classification accuracy of SVM has reached more than $99.5 \%$. Generally, post-processing can remove the isolated points, and further improve the classification accuracy. However, different approaches apply to different images, post-processing is not universally applicable, in some cases the classification accuracy may also fall. As for ML, the classification accuracy of ML-C and ML-S is less than ML to a certain extent. Therefore, in the process of classification, image processing methods should be selected reasonably combined with the image features.

Table 3 Classification accuracy

\begin{tabular}{cccc}
\hline $\begin{array}{c}\text { Classification } \\
\text { results }\end{array}$ & $\begin{array}{c}\text { Classification } \\
\text { accuracy/\% }\end{array}$ & Kappa coefficient & Reference pixels \\
\hline SVM & 99.1108 & 0.9886 & 10589 \\
SVM-M & 99.5039 & 0.9936 & 10631 \\
SVM-C & 99.2699 & 0.9906 & 10606 \\
SVM-S & 99.1108 & 0.9886 & 10631 \\
ML & 97.6507 & 0.9698 & 10433 \\
ML-M & 97.8192 & 0.9720 & 10451 \\
ML-C & 97.5384 & 0.9684 & 10421 \\
ML-S & 97.6413 & 0.9697 & 10684 \\
\hline
\end{tabular}

Table 4 is the distribution of the farmland objects. From the table we can obtain the distribution and proportion of different objects. In this paper, the proportion of crops, roads, trees, soil, and background were $27.5 \%, 13.1 \%, 35.8 \%, 14.1 \%$, and $9.6 \%$, among which the largest proportion is trees, followed by the crops. Therefore, knowing the distribution of farmland objects through classification is helpful for field work of intelligent agricultural robots, and ultimately it can achieve better perception for environment information.

Table 4 Distribution of farmland objects

\begin{tabular}{cccccc}
\hline Farmland objects & Crop & Road & Trees & Soil & Background \\
\hline Distribution pixels & 35160 & 18304 & 45754 & 16781 & 12271 \\
$\begin{array}{c}\text { Distribution } \\
\text { proportion/\% }\end{array}$ & 27.469 & 13.110 & 35.745 & 14.089 & 9.587 \\
\hline
\end{tabular}

\section{Conclusions}

Taking near-infrared farmland hyper-spectral images as research objects, this research proposed a distance-based method to determine the separability of ROI, so as to realize the classification of farmland objects. At first, the preprocessing of image correction and de-noising was conducted. Then, the corresponding features of the farmland objects were extracted, and maximum likelihood and support vector machine (SVM) were used for classification. Finally, the classification results were analyzed after post-processing. Experimental results showed that the total classification accuracy reached more than 97\%, and the distance-based determination method can better extract the feature values using the proposed methods. At the same time, composing hyper-spectral imaging technology and spectral analysis technology can achieve farmland objects extraction. Therefore, the research can provide a basis for the research of outdoor farmland near-infrared hyper-spectral images.

Actually, the background is too complex and the farmland objects themselves have fuzzy boundaries. The classification results and accuracy could be affected to a certain degree. For intensive study, segmentation before feature extraction for the hyper-spectral images can be a future research direction. The acquisition of the training samples would spend a great deal of effects, and automatic access to the training samples could be a broadening area. In the process of identification, only the defined classes acquired in the training process can be identified. While, the undefined classes cannot be recognized, which need further researches.

\section{Acknowledgements}

This work is supported by the Shaanxi Key Laboratory of Complex System Control and Intelligent Information Processing under Grant No. 2016CP01, Xi'an University of Technology, Xi'an Science and Technology Plan Projects under Grant No. NC1504 (2), the National Natural Science Foundation of China under Grant No. 31101075, the National High Technology Research and Development of China (863 Program) 
under Grant No. 2013AA10230402, Natural Science

Fundamental Research Plan of Shaanxi Province under Grant No.2016JM6038, Fundamental Research Funds for the Central Universities, NWSUAF, China, Grant No. 2452015060 .

\section{[References]}

[1] Zhu J, Wang Y, Yu H, Xu H. Mobile robot autonomous perceiving slope terrain under unknown environment. Chinese Journal of Scientific Instrument, 2010; 31(8): 1916-1920. (in Chinese)

[2] Zhang R B, Yi L L, Gu H W. Environment perception for AUV in uncertain ocean environment. Journal of Computer Research and Development, 2013; 50(9): 1981-1991.

[3] Dan J J, Peng Y K, Wang W, Li Y Y, Wu J H, Zhang L L. Simultaneous detection of external and internal quality parameters of apples using hyperspectral technology. Transactions of the CSAM, 2011; 42(3): 140-144. (in Chinese)

[4] Wang C. Study on compression algorithm of hyper-spectral image. Master's thesis. Nanjing University of Science and Technology, 2014. (in Chinese)

[5] ElMasry G, Wang N, Vigneault C. Detecting chilling injury in red delicious apple using hyper-spectral imaging and neutral networks. Postharvest Biology and Technology, 2009; 52: 1-8.

[6] Cai J R, Wang J H, Huang X Y, Chen Q S. Detection of rust in citrus with hyperspectral imaging technology. OPTO-Electronic Engineering, 2009; 36(6): 26-30.

[7] Cheng G S, Guo J X, Atimu R, Kang Y, Shi Z. The size detection of apple using hyperspectral imaging technology. Journal of Agricultural Mechanization Research, 2012; 34(6): 108-112. (in Chinese)

[8] Ghamisi P, Benediktsson J, Ulfarsson M. Spectralcspatial classification of hyperspectral images based on hidden markov random fields. IEEE Transactions on Geoscience and Remote Sensing, 2013; 52(5): 2565-2574.

[9] Liao W Z, Pizurica A, Scheunders P, Philips W, Pi Y. Semisupervised local discriminant analysis for feature extraction in hyperspectral images. IEEE Transactions on Geoscience and Remote Sensing, 2012; 51(1): 184-198.

[10] Tarabalka Y, Fauvel M, Chanussot J, Benediktsson J A. SVM and MRF-based method for accurate classification of hyperspectral images. IEEE Transactions on Geoscience and Remote Sensing, 2010; 7(4): 736-740.

[11] Tarabalka Y, Chanussot J, Benediktsson J A. Benediktsson. Segmentation and classification of hyperspectral images using watershed transformation. Pattern Recognition, 2010;
43(7): 2367-2379.

[12] Luo G, Chen G, Tian L, Qin K, Qian S E. Minimum noise fraction versus principal component analysis as a preprocessing step for hyperspectral imagery denoising. Canadian Journal of Remote Sensing, 2016; 42(2): 106-116.

[13] Dong L F. Research on hyper-spectral preprocessing. Master's thesis. Changan University, 2007.

[14] Boardman J, Kruse F. Automated spectral analysis: a geological example using AVIRIS data, north grapevine mountains, Nevada. Surgical Endoscopy, 1994; 1(1): 407-418.

[15] Si B L. An image retrieval method based on regions of interest. Master's thesis. Institute of Computing Technology, Chinese Academy of Sciences, 2002. (in Chinese)

[16] Zhou J L., Khawaja M A, Li Z D, Sun J J, Wang Y, Chen F. Making machine learning useable by revealing internal states update: A transparent approach. International Journal of Computational Science and Engineering, 2016; 4(13): 378-389.

[17] Brendel B, von Teuffenbach M, Noël P B, Pfeiffer F, Koehler T. Penalized maximum likelihood reconstruction for X-ray differential phase-contrast tomography. Medical Physics, 2016; 43(1): 188-194.

[18] Varma M K S, Rao N K K, Raju K K, Varma G P S. Pixel-based classification using support vector machine classifier. International Conference on Advanced Computing. IEEE, 2016.

[19] Liu H P, An H J. Maximum likelihood in identifying Huhhot greening tree species. Journal of Northeast Forestry University, 2014; 42(7): 157-160.

[20] Zhang H Y, Peng Y K, Wang W, Zhao S W, Liu Q Q. Rapid nondestructive detection of water content in fresh pork based on spectroscopy technique combined with support vector machine. Spectroscopy and Spectral Analysis, 2012; 32(10): 2794-2798.

[21] Li W, Hu B, Wang M W. Discrimination of varieties of borneol using terahertz spectra based on principal component analysis and support vector machine. Spectroscopy and Spectral Analysis, 2014; 34(12): 3235-3240.

[22] Ma X, Zhang Y, Wang Y. Performance evaluation of kernel functions based on grid search for support vector regression. International Conference on Cybernetics and Intelligent Systems, 2015; pp.283-288.

[23] Liu Z, Zuo M J, Zhao X, Xu H. An analytical approach to fast parameter selection of Gaussian RBF Kernel for support vector machine. Journal of Information Science \& Engineering, 2015; 31(2): 691-710.

[24] Wong $\mathrm{T}$ T. Performance evaluation of classification algorithms by $\mathrm{k}$-fold and leave-one-out cross validation. Pattern Recognition, 2015; 48(9): 2839-2846. 